\title{
A Large Amplitude Flaring dMe Star in the 1978 October 6B $\gamma$-ray Burst Error Box ${ }^{\star}$
}

\author{
J. Greiner ${ }^{1}$, C. Motch ${ }^{2}$ \\ 1 Max-Planck-Institut für Extraterrestrische Physik, D-85740 Garching, Germany \\ 2 CNRS, UA 1280, Observatoire de Strasbourg, 11 rue de l'Université, \\ F-67000 Strasbourg, France
}

\section{Introduction}

During a search for flaring optical counterparts to $\gamma$-ray burst sources (GRBs) on archival patrol plates, an optical outburst image was found in the $3200 \mathrm{arcmin}^{2}$ error box of the GRB which occurred on 1978 October 6 (GB 781006B = GBS $0008+13$; Greiner et al. 1991) and was named S 10933 according to the convention of new Sonneberg variables. This outburst image appears on three simultaneously exposed plates taken on 1966 August $14 / 15$ from 23.33 to 0.13 UT. The brightness estimates yielded $m_{\mathrm{pg}}=13^{\mathrm{m}} 3 \pm 0^{\mathrm{m}} 1$ and $m_{v}=13^{\mathrm{m}} .8 \pm 0.2$ with a high negative colour index of $\mathrm{C}=-0, \mathrm{~m}^{\mathrm{m}} \pm 0 . \mathrm{m}$.

The coordinates of these images (measured on the Tessar plate $\mathrm{Te}_{3}$ 5154) are (equinox 1950.0) $\alpha=0^{\mathrm{h}} 10^{\mathrm{m}} 07^{8} 6, \delta=+12^{\circ} 51^{\prime} 24^{\prime \prime}\left(1 \sigma\right.$ error of $\left.\pm 2^{\prime \prime}\right)$. Within the $3 \sigma$ error circle of the position of the object a star of $m_{B}=18$ is visible on the Palomar Observatory Sky Survey prints. Here, we report spectroscopic observations of this faint object.

\section{Observations and Results}

CCD B and R images of the field of the optical transient were obtained from 1991 November 10 to 12 with the $1.2 \mathrm{~m}$ telescope at Observatoire de Haute-Provence (OHP). Low and medium resolution spectra of the brightest optical object A were obtained on 1992 February 4, 7 and 8 with the CARELEC spectrograph attached at the OHP $1.93 \mathrm{~m}$ telescope. Further spectroscopic observation were obtained using the $3.5 \mathrm{~m}$ telescope at Calar Alto, equipped with the Cassegrain spectrograph, on 1992 Sep 28 - Oct 1 . The slit was put parallel to the row of faint objects apparently aligned with the bright candidate. On $1992 \mathrm{Sep} 30$, three spectra with a grating of $60 \AA / \mathrm{mm}$ were acquired.

\footnotetext{
* Partly based on observations collected at the German-Spanish Astronomical Centre, Calar Alto, operated by the MPI für Astronomie, Heidelberg, jointly with the Spanish National Commission for Astronomy, and on observations obtained with the $1.2 \mathrm{~m}$ and $1.9 \mathrm{~m}$ telescopes of the Observatoire de Haute-Provence, CNRS, France.
} 
Table 1. Other large amplitude flares

\begin{tabular}{lcl}
\hline Star & $\Delta m_{B}(\mathrm{mag})$ & References \\
\hline CZ Cnc & $\approx 9.5$ & Lovas 1977, Schaefer 1990, Greiner \& Motch 1994 \\
AF Psc & $\approx 7.5$ & Bond 1976, Greenstein 1977 \\
UV Cet & $\approx 6.5$ & Jarrett \& Gibson 1975 \\
EV Lac & $\approx 6.0$ & Roizman \& Shevchenko 1982 \\
AM Her (comp) & $\approx 5.9$ & Shakhovskoy et al. 1993 \\
S 10933 & $\geq 5.2$ & this work, Greiner \& Motch 1995 \\
AD Leo & $\approx 4.1$ & Hawley \& Pettersen 1991 \\
G102-21 & $\approx 3.9$ & Pagano et al. 1995 \\
\hline
\end{tabular}

The spectral type of the central object was estimated by comparing our flux calibrated spectra with spectra in electronic atlases (Jacoby et al. (1984) and Turnshek et al. (1985)). We conclude that the central star is a M3-M5e late type dwarf. The lack of Li absorption tells us that the star is older than $\approx 10^{7} \mathrm{yr}$ (Rebolo 1991). Comparison with photometric standards in NGC 7790 (Christian et al. 1985) yielded $B=18.50 \pm 0.20$.

Two additional objects lie within or slightly outside the $3 \sigma$ error circle of the archival flare event. These are at least $2^{\mathrm{m}}$ fainter than the dMe star. They are aligned southeastwards from the central $\mathrm{dMe}$ star together with a third one which is already outside the $3 \sigma$ position. Our long slit spectra of the three aligned objects show that the brightest notch (object $D$ ) located at the end of the apparent alignment is an $M$ star, however without noticeable Balmer emission. No usable spectra could be obtained from notches $B$ and $C$ located in between the $\mathrm{dMe}$ star and object $\mathrm{D}$. These objects exhibit flat continua without recognizable emission or absorption features.

\section{Discussion}

\subsection{The flare star}

Our observations leave no doubt that the bright dMe star is responsible for the large optical flare of 1966. The spectral type indicates an absolute magnitude $M_{V}$ in the range of 10 to 14 and a distance of 40 to $250 \mathrm{pc}$. The apparent magnitudes during the flare and in quiescence translate into visual luminosities (blue band) of $4 \times 10^{31}(d / 100 \mathrm{pc})^{2} \mathrm{erg} / \mathrm{s}$ and $3.5 \times 10^{29}(d / 100 \mathrm{pc})^{2} \mathrm{erg} / \mathrm{s}$, respectively.

Assuming that the flare lasted as long as (or longer than) the exposure time of the discovery plates $(40 \mathrm{~min})$, implies a peak amplitude of about $\Delta m_{B} \approx 5{ }^{\mathrm{m}} 2$. Alternatively, if the event belongs to the class of fast flares with typical duration shorter than $\approx 10$ minutes, the peak amplitude could have been as high as $7-9^{\mathrm{m}}$. For comparison, other large amplitude flaring stars are listed in Table 1. Even a peak amplitude of $5 \cdot 2$ (corresponding to a flare energy release of $10^{35}(d / 100$ pc) $)^{2}$ erg in the optical bandpass) are rare for a flare star and we can conclude that the event witnessed in 1966 was certainly unusual. 


\subsection{The relation to GBS $0008+13$}

At the time of the discovery (Greiner et al. 1991) it was assumed that the recorded optical flash was a likely counterpart candidate for a GRB. Indeed, this optical flash is well positioned within a $3200 \mathrm{arcmin}^{2}$ error box of a GRB which occured on October 6, 1978 (GB 781006B = GBS 0008+13, Atteia et al. 1987). The rather extreme value for the flare amplitude might favour speculations whether or not this flare is indeed related to a GRB. In fact, the possible relation with stellar flares was among the first models proposed to explain GRBs. Nearby flare stars are isotropic in the sky and have a $-3 / 2$ slope in their $\log N-\log S$ distribution (for a sampling distance smaller than $\approx 300 \mathrm{pc}$ ) and thus meet one of the statistical GRB criteria. Recently, Liang and Li (1993) have shown that flare stars could account for as many as half of the BATSE triggers without violating the observed $\log N-\log S$ distribution of GRBs.

Estimating the a priori probability of discovering such a large amplitude flaring star in a rather large $\left(3200 \operatorname{arcmin}^{2}\right) \gamma$-ray error box is difficult because of the various statistical biases in flare star catalogs and flare observational data bases. There are convincing evidences that flare activity preferentially occurs in rather young stars (e.g. Mirzoyan 1990). Most flaring $M$ stars exhibit strong Balmer emission (Pettersen 1991) while kinematical studies of dMe stars show that they belong to the young disk population with a mean age of $\approx 2 \times 10^{9}$ yrs (Giampapa \& Liebert 1986). However, old stars in close binaries may also exhibit strong flaring activity (Haisch et al. 1991).

Using the stellar population model of Robin \& Crézé (1986) which explicitly handles the age parameter, we predict that $\approx 10$ stars with $B \leq 18.5$ and younger than $10^{9}$ yrs should be present inside the GRB error box, including two late $M$ type dwarf stars. Therefore, in the absence of stronger constraints on the age of the Me dwarf and reliable statistics on flare amplitudes, we cannot prove that this particular flare star has caused the $\gamma$-ray burst GB $781006 \mathrm{~B}$, nor can we dismiss the flare star explanation for this particular GRB.

\section{Summary}

We have identified the 1966 optical flash to be caused by a $B=18^{\mathrm{m}} 5 \mathrm{mag} \mathrm{dMe}$ flare star. We think that the relation between this flare star and the $\gamma$-ray burst source GBS $0008+13$ is possible but unlikely. Even in this case of an unique archival object observed on synchronously exposed plates inside a GRB error box we are still left with an explanation other than a $\gamma$-ray burst optical counterpart.

Our identification confirms the feasibility of searching archival plate collections for real optical flashes of astrophysical origin. Unfortunately, the existence of an unknown number of such large amplitude flaring stars as the one reported here poses serious problems for wide-field GRB optical flash searches. More details can be found in Greiner \& Motch (1995).

Acknowledgements: JG is partly supported by the Deutsche Agentur für Raumfahrtangelegenheiten (DARA) GmbH under contract number FKZ 50 OR 9201. CM acknowledges support from a CNRS-MPG cooperation contract. 


\section{References}

Atteia J.-L., Barat C., Hurley K., Niel M., Vedrenne G., Evans W.D., Fenimore E.E., Klebesadel R.W., Laros J.G., Cline T., Desai U., Teegarden B., Estulin I.V., Zenchenko V.M., Kuznetsov A.V., Kurt V.G., 1987, ApJS 64, 305

Bond H.E., 1976, IBVS 1160

Christian C.A., Adams M., Barnes J.V., Butcher H., Hayes D.S., Mould J.R., Siegel M., 1985, PASP 97, 363

Giampapa M.S., Liebert J., 1986, ApJ 305, 784

Greenstein J.L., 1977, PASP 89, 304

Greiner J., Naumann Ch., Wenzel W., 1991, A\&A 242, 425

Greiner J., Motch C., 1994, IBVS 4092

Greiner J., Motch C., 1995, A\&A 294, 177

Haisch B., Strong K.T., Rodono M., 1991, ARA\&A 29, 275

Hawley S.L., Pettersen B.R., 1991, ApJ 378, 725

Jacoby G.H., Hunter D.A., Christian C.A., 1984, ApJS 56, 257

Jarrett A.H., Gibson J.B., 1975, IBVS 979

Liang E.P., Li H., 1993, A\&A 273, L53

Lovas M., 1977, IBVS 1345

Mirzoyan L.V., 1990, IAU Symp 137, L.V. Mirzoyan, B. Pettersen \& M.K. Tsvetkov (eds.), Kluwer, Dordrecht, p. 1

Pettersen B.R., 1991, Mem. S.A. It. 62, 217

Pagano I., Ventura R., Rodonò M., Peres G., Micela G., 1995, this volume p. 95

Rebolo R., 1991, in IAU Symp 145, Evolution of Stars: The Photospheric Abundance Connection, G. Michaud \& A. Tutukov (eds.), p. 85

Robin A., Crézé M., 1986, A\&A 157, 71

Roizman G.Sh., Shevchenko V.S., 1982, Sov. Astron. Lett. 8, 85

Schaefer B.E., 1990, ApJ 353, L25

Shakhovskoy N.M., Alexeev I.Yu., Andronov I.L., Kolesnikov S.V., 1993, in Cataclysmic variables and related physics, O. Regev \& G. Shaviv (eds.), Inst. Phys. Publ., Bristol, p. 237

Turnshek D.E., Turnshek D.A., Craine E.R., Boeshaar P.C., 1985, An Atlas of Digital Spectra of Cool Stars, Western Research Company, Tucson

H. Zinnecker: Have any of the large amplitude flare stars that you have listed been observed with ROSAT pointed observations, and if so, have any been detected?

J. Greiner: The objects CZ Cnc, AF Psc, and S 10933 are not covered by ROSAT pointed observations. The other flare stars of Tab. 1 are all observed by ROSAT and to my knowledge are also detected (quiescent emission).

D. Dravins: What accuracy in $\gamma$-ray burst positions will be required in order for flare stars not to contribute any significant background in searches for optical counterparts?

J. Greiner: With the present number density of known flare stars (outside of stellar clusters) one needs $1 \mathrm{arcmin}^{2}$ sized error boxes to push the flare star contamination below $0.1 \%$. However, discoveries of quite a substantial number of new flare stars even in the galactic field (see e.g. Richter et al. 1995, these proceedings, p. 69), make this estimate an upper limit. 